\title{
Level set simulations of turbulent thermonuclear deflagration in degenerate carbon and oxygen
}

W. Schmidt, Lehrstuhl für Astronomie, Universität Würzburg, Am Hubland, D-97074 Würzburg, Germany

'W. Hillebrandt, Max-Planck-Institut für Astrophysik, Karl-Schwarzschild-Str. 1, D-85741 Garching, Germany

J. C. Niemeyer, Lehrstuhl für Astronomie, Universität Würzburg, Am Hubland, D-97074 Würzburg, Germany

(Received 00 Month 200x; In final form 00 Month 200x)

"

We study the dynamics of thermonuclear flames propagating in fuel stirred by stochastic forcing.

The fuel consists of carbon and oxygen in a state which is encountered in white dwarfs close to the Chandrasekhar limit. The level set method is applied to represent the flame fronts numerically. The computational domain for the numerical simulations is cubic, and periodic boundary conditions are imposed. The goal is the development of a suitable flame speed model for the small-scale Odynamics of turbulent deflagration in thermonuclear supernovae. Because the burning process in a supernova explosion is transient and spatially inhomogeneous, the localised determination of subgrid scale closure parameters is essential. We formulate a semi-localised model based on the dynamical equation for the subgrid scale turbulence energy $k_{\mathrm{sgs}}$. The turbulent flame speed $s_{\mathrm{t}}$ is of the order $\sqrt{2 k_{\mathrm{sgs}}}$. In particular, the subgrid scale model features a dynamic procedure for the calculation of the turbulent energy transfer from resolved toward subgrid scales, which has been successfully applied to combustion problems in engineering. The options of either including or suppressing inverse I energy transfer in the turbulence production term are compared. In combination with the piece-wise Parabolic method for the hydrodynamics, our results favour the latter option. Moreover, different choices for the constant of proportionality in the asymptotic flame speed relation, $s_{\mathrm{t}} \propto \sqrt{2 k_{\mathrm{sgs}}}$, are investigated.

Keywords: Combustion, thermonuclear, turbulence, large eddy simulation, level set method

\section{Introduction}

A certain kind of stellar explosion, known as type Ia supernovae among astronomers, is currently explained by the thermonuclear explosion of an electron-degenerate stellar remnant 1]. Such an object, which is called a white dwarf, emanates from the burn-out of stars comparable in mass to our Sun and is mainly composed of carbon and oxygen. If the white dwarf has a companion star in close orbit, it can grow by accreting material from the companion. Under certain conditions, the white dwarf's mass will steadily increase and finally approach the Chandrasekhar limit, which is the maximal mass that can 
be supported by the degeneracy pressure of electrons 2]. As the temperature and density are increasing, thermonuclear burning of carbon and oxygen gradually sets in. Close to the Chandrasekhar mass, the conditions in the core of the white dwarf eventually pass a critical threshold [3. At this point, the rate of thermonuclear reactions rises rapidly, and a runaway is initiated, which incinerates and disrupts the whole star within a few seconds. The total energy release is of the order $10^{51} \mathrm{erg}$ [4].

The thermonuclear combustion of degenerate carbon and oxygen of density in the range $\sim 10^{7} \ldots 10^{9} \mathrm{~g} \mathrm{~cm}^{-3}$ proceeds in the form of a deflagration [5], 6]. Since the nuclear ash produced by the burning process has less specific weight than the surrounding unprocessed material, it becomes Rayleigh-Taylor unstable. Since turbulence is subsequently produced, the flames get corrugated and folded [7, 8. In consequence, there is a positive feedback mechanism of turbulence enhancing the burning and, according to state-of-the-art numerical simulations, eventually results in an explosion [9, 10, 11. Although it cannot be ruled out that a transition from the deflagration to a detonation might set in at some stage 12,13, turbulent deflagration plays a crucial role in the theoretical modelling of thermonuclear supernovae in any case.

The subject of this article is the dynamics of flame fronts on length scales much smaller than the size of a Chandrasekhar-mass white dwarf. To that end, an artificial scenario was set up. A turbulent flow is produced by means of stochastic stirring in a cubic domain subject to periodic boundary conditions 14,15. Thermonuclear burning is ignited in small spherical regions and subsequently evolved by means of the level set method [16 17. The complicated network of thermonuclear reactions encountered in a type Ia supernova is substituted by the effective fusion of equal mass fractions of ${ }^{12} \mathrm{C}$ and ${ }^{16} \mathrm{O}$ to ${ }^{56} \mathrm{Ni}$ and ${ }^{4} \mathrm{He}$ as representative reaction 18 . The equation of state is dominated by the degenerate gas of relativistic free electrons. Thus, the approximate relation $P \propto \rho^{4 / 3}$ applies, while the temperature has virtually no influence on the pressure. This is actually the reason for the runaway, because the negative feedback between heating and expansion in non-degenerate matter is absent. The exact equation of state has no analytic solution and must be integrated numerically. Moreover, contributions from nuclei, photons and pair electron-positron pair creation at temperatures of the order $10^{10} \mathrm{~K}$ are taken into account (section 3.2 of [19]). The fluid dynamics is treated by means of the piece-wise parabolic method (PPM) within the framework of the Euler equations [20].

In the corrugated flamelet regime of combustion, the flame propagation is affected by turbulence on length scales ranging from the Gibson length up to the integral length scale 21. In general, only the the largest length scales can be resolved in a numerical simulation. In order to account for the wrinkling of the flame surface on length scales smaller than the cutoff scale of a simulation, an effective propagation speed, the so-called turbulent flame speed, must be 
calculated. This involves a subgrid scale (SGS) model for the local budget of turbulence energy contained in numerically unresolved modes. In this article, we present a numerical study in which different variants of the SGS turbulence energy model are compared (section 4.3 in 22]). In particular, we adopted a dynamical procedure for the computation of SGS closure parameters. This procedure was proposed by Kim and Menon for the application in LES of gas turbine combustor flows [23].

\section{The physics of turbulent thermonuclear deflagration}

The mechanism of deflagration is based on thermal conduction, as opposed to a detonation, which proceeds via shock compression. Unburned material (fuel) is heated in the vicinity of the reaction zone and thereby gets ignited. Once heat generation is balanced by diffusion, the burning zone is propagating at a steady subsonic speed, and pressure equilibrium is maintained across the reaction zone. Basically, this characterises what is commonly known as a flame. For chemical combustion, a distinction is made between premixed and diffusive flames. Thermonuclear flames are trivially premixed, because no additional agent, like oxygen in most chemical burning processes, is required. The local propagation speed of the flame, which is solely determined by microscopic properties, is called the laminar burning speed. The notion of a flame applies, if fluid motions do not significantly disturb the burning process within the reaction zone, i. e., the characteristic time scale of burning is much smaller than the kinetic time scale of velocity fluctuations on length scales comparable to the flame thickness $\delta_{\mathrm{F}}$. Equivalently, $\delta_{\mathrm{F}} \ll l_{\mathrm{G}}$, where the Gibson length scale $l_{\mathrm{G}}$ is the smallest length scale on which the burning process is affected by fluid motion [21. The condition $\delta_{\mathrm{F}} \ll l_{\mathrm{G}}$ thus specifies the flamelet regime of combustion, which is reviewed in this section. For the thermonuclear combustion in $\mathrm{C}+\mathrm{O}$ white dwarfs, it appears that the flamelet description is valid for $\rho \gtrsim 3 \cdot 10^{7} \mathrm{~g} \mathrm{~cm}^{-3}$ [24. In thermonuclear supernovae, most of the burning takes place at significantly higher densities.

\subsection{Laminar burning}

The width of the reaction zone, $\delta_{\mathrm{F}}$, is determined by the equilibrium between energy generation due to nuclear reactions and the rate of diffusion caused by thermal conduction ( $\S 128$ in [25]). The balance between these processes can be expressed in terms of their characteristic time scales, $\tau_{\text {burn }}$ and $\tau_{\text {cond }}$. The former is given by $\tau_{\text {burn }} \sim \rho \varepsilon_{\text {nuc }} / B$, where $\varepsilon_{\text {nuc }}$ is the energy generated by the fusion of a unit mass of nuclear fuel, and $B$ is the rate of energy release per unit volume. The time scale of conduction, on the other hand, can be 
expressed as $\tau_{\text {cond }} \sim \delta_{\mathrm{F}}^{2} / l_{\mathrm{e}} c$, where $l_{\mathrm{e}}$ is the mean free path of the electrons, which contribute the major part of the thermal conductivity, and $c$ is the speed of sound. Setting these two time scales equal, one finds that the flame thickness $\delta_{\mathrm{F}}$ is approximately given by

$$
\delta_{\mathrm{F}} \sim \sqrt{\frac{\rho \varepsilon_{\mathrm{nuc}} l_{\mathrm{e}} c}{B}} .
$$

Defining the laminar flame speed by $s_{\text {lam }}=\delta_{\mathrm{F}} / \tau_{\text {burn }}$, we have

$$
s_{\mathrm{lam}} \sim \sqrt{\frac{l_{\mathrm{e}} c B}{\rho \varepsilon_{\mathrm{nuc}}}} .
$$

The specific energy release for the fusion of ${ }^{12} \mathrm{C}$ and ${ }^{16} \mathrm{O}$ to ${ }^{56} \mathrm{Ni}$ is $\varepsilon_{\text {nuc }} \approx$ $7 \cdot 10^{17} \mathrm{erg} \mathrm{g}^{-1}$ [18. The flame speed $s_{\text {lam }}$ for the thermonuclear combustion of degenerate carbon and oxygen was computed numerically for a wide range of mass densities and nuclear compositions by Timmes and Woosley [6]. For example, $s_{\text {lam }} \approx 3.6 \cdot 10^{6} \mathrm{~cm} \mathrm{~s}^{-1}$ and $\delta_{\mathrm{F}} \approx 2.9 \cdot 10^{-4} \mathrm{~cm}$ for equal mass fractions of carbon and oxygen at a density $10^{9} \mathrm{~g} \mathrm{~cm}^{-3}$.

\section{$2.2 \quad$ Turbulent burning}

So far, we have only been concerned with the microphysics of thermonuclear deflagration. Let us now consider the combustion of $\mathrm{C}+\mathrm{O}$ fuel in a state of turbulent motion. For brevity, we shall assume the case of steady isotropic turbulence, i.e. a statistically self-similar hierarchy of vortices or eddies. Each vortex of size $l$ has a characteristic velocity $v^{\prime}(l)$ and an associated turn-over time $\tau_{\text {eddy }}(l)=l / v^{\prime}(l)$. If $v^{\prime}(l)$ is small compared to the laminar flame speed $s_{\text {lam }}$, then the flame front will propagate through a region of size $l$ in a time much faster than the turn-over time $\tau_{\text {eddy }}(l)$. Hence, the turbulent flow appears to be more or less "frozen" with respect to the burning process on these scales. For $v^{\prime}(l) \gg s_{\text {lam }}$, on the other hand, the front is significantly distorted while it is crossing a vortex of diameter $l$. Hence, there is a threshold scale on which burning decouples from turbulence. This is the Gibson length $l_{\mathrm{G}}$, which is defined by 24

$$
v^{\prime}\left(l_{\mathrm{G}}\right)=s_{\text {lam }} .
$$

At length scales $l \gg l_{\mathrm{G}}$, turbulence corrugates the flame and thereby increases its surface area. Consequently, turbulence enhances the burning process and the release of heat is growing. This can be accounted for by introducing a 
turbulent propagation speed $s_{\mathrm{t}}(l)$. In other words, averaging over regions of size $l$, the flame front propagates with an effective speed $s_{\mathrm{t}}(l)$ greater than the laminar burning speed $s_{\text {lam }}$, which specifies the local speed of any portion of the flame smaller than $l_{\mathrm{G}}$.

For $l \gg l_{\mathrm{G}}, s_{\mathrm{t}}(l)$ becomes asymptotically independent of $s_{\mathrm{lam}}$. The fundamental hypothesis applied in this article is that $s_{\mathrm{t}}(l)$ is then given by the magnitude of the turbulent velocity fluctuations $v^{\prime}(l)$. In the framework of the phenomenological Kolmogorov theory of isotropic turbulence [26], this velocity obeys the scaling law $v^{\prime}(l) \propto l^{1 / 3}$ in the inertial subrange, i.e. in the range of length scales which are neither affected by the viscosity of the fluid nor large-scale energy injection. Therefore,

$$
s_{\mathrm{t}}(l) \sim v^{\prime}(l) \propto l^{1 / 3}
$$

The relation $s_{\mathrm{t}}(l) \sim v^{\prime}(l)$ was first proposed by Damköhler, who studied Bunsen cones in the laboratory [27. Further validation of this conjecture came from numerical studies [28. A motivation based on a theoretical analysis in the framework of the level set prescription was given by Peters [29].

\section{The numerical modelling of turbulent flame propagation}

In early studies of thermonuclear deflagration [7,9], a reactive-diffusive flame model with artifical diffusion and reaction rates was applied. In this approach, the thickness of the flame is artifically increased over several grid cells and the propagation speed is adjusted to a prescribed value. On the other hand, the level set method proposed by Osher and Sethian [16,31] is a front tracking method which describes the interface separating ash from fuel as a genuine discontinuity. The interface is numerically represented by the set of all points for which a suitably chosen distance function vanishes, i.e. the zero level set. This is a sensible approximation if the physical flame thickness is very small compared to the Gibson scale. For the simulation of thermonuclear combustion in type Ia supernovae, the level set method was implemented by Reinecke [17, 19.

\subsection{The level set method}

Let $G(\boldsymbol{x}, t)$ be a signed distance function with the property $|\nabla G|=1$. The absolute value $|G(\boldsymbol{x}, t)|$ is equal to the minimal distance of the point $\boldsymbol{x}$ from the flame front at time $t$. The front itself is given by the constraint $G(\boldsymbol{x}, t)=0$, i.e. it is represented by the zero level set $\Gamma(t)=\{\boldsymbol{x} \mid G(\boldsymbol{x}, t)=0\}$. With the sign convention $G(\boldsymbol{x}, t)>0$ in regions containing burned material, the unit normal 
vector pointing towards unburned material is given by $\boldsymbol{n}=-\boldsymbol{\nabla} G /|\boldsymbol{\nabla} G|$. The time evolution of the front $\Gamma(t)$ is implicitly determined by the total time derivative of $G\left(\boldsymbol{x}_{\Gamma}(t), t\right)=0$. For a certain point at the front, $\boldsymbol{x}_{\Gamma}(t) \in \Gamma(t)$, we have

$$
\frac{\mathrm{d}}{\mathrm{d} t} G\left(\boldsymbol{x}_{\Gamma}(t), t\right)=\frac{\partial G}{\partial t}+\dot{\boldsymbol{x}}_{\Gamma} \cdot \nabla G=0
$$

The speed function $\dot{\boldsymbol{x}}_{\Gamma}$ is given by the sum of two contributions. Firstly, the advection speed normal to the flame front, $\boldsymbol{v}_{\mathrm{u}} \cdot \boldsymbol{n}$, where $\boldsymbol{v}_{\mathrm{u}}$ is the velocity of the fuel immediately ahead of the front in an Eulerian frame of reference. And, secondly, the intrinsic propagation speed $s$ of the flame front relative the fuel.

The local equation (5) can be formulated globally as well, without constraining the position $\boldsymbol{x}$ to the flame surface. Substituting the definition of the normal vector $\boldsymbol{n}$ and expressing the speed function in the form $\boldsymbol{v}_{\mathrm{u}} \cdot \boldsymbol{n}+s$, the evolution equation for the level set function at any point in space becomes

$$
\frac{\partial G(x, t)}{\partial t}=\left[\boldsymbol{v}_{\mathrm{u}}(x, t)+s(x, t) \boldsymbol{n}(x, t)\right]|\boldsymbol{\nabla} G(x, t)| .
$$

The advection part on the right-hand side can be treated with a finite-volume scheme, for instance, the PPM. The intrinsic front propagation is usually calculated by means of an entropy-satisfying upwind scheme. In general, non-planar fronts will develop sharp corners and the corresponding level set must be a weak solution: Information about the initial conditions is lost, once a cusp has formed, and the subsequent evolution is irreversible. The corresponding entropy condition can be formulated in the following way: Once a certain fluid element is burned, it remains burned thereafter. In fact, this implies the equivalent Huyghen's principle in optics for the propagation of the front over an infinitesimal interval of time (section 5 in [31]). Finally, in order to preserve the property $|\nabla G|=1$, the updated distance function has to be corrected after each time step. In the implementation of Reinecke, this is achieved by means of re-initialisation [17.

For the complete implementation of the level set technique, both the burned and the unburned state in an intersected numerical cell must be reconstructed from the jump conditions across the front. Assuming that there is a volume fraction of unburned material $\alpha$, conservation of momentum imposes the constraint

$$
\rho \boldsymbol{v}=\alpha \rho_{\mathrm{u}} \boldsymbol{v}_{\mathrm{u}}+(1-\alpha) \rho_{\mathrm{b}} \boldsymbol{v}_{\mathrm{b}}
$$

given the finite-volume averages $\rho$ and $\boldsymbol{v}$. The volume fraction $\alpha$ can be calculated by linearly interpolating the discrete numerical values of the distance 
function $G$. Supplementing the momentum equation with the Rayleigh criterion, the Hugoniot jump condition and the continuity constraint for the tangential velocity components, a non-linear system of equations is obtained. The solution yields $\boldsymbol{v}_{\mathrm{u}}, \boldsymbol{v}_{\mathrm{b}}$ and the corresponding state variables (cf. 32]). This procedure of in-cell reconstruction was indeed successfully implemented for chemical combustion problems [33. However, the deviation of the interpolated front element from the exact smooth solution can introduce significant errors in the reconstructed states. In particular, it is sometimes impossible to reconstruct physically sound states for degenerate matter, because of the stiffness of the equation of state. Moreover, one faces topological ambiguities for certain configurations. A pragmatic method is to average over all possible values, whenever one of these rare cases is encountered [17. Although Röpke et al. have recently succeeded with the implementation of in-cell reconstruction for the problem of thermonuclear flame propagation in two dimensions 32, generalising the algorithm to three dimensions would be much more challenging.

The difficulties outlined above are avoided with the so-called passive implementation, where the difference between burned and unburned states is neglected and the advection speed is set equal to $\boldsymbol{v} \cdot \boldsymbol{n}$. The discrete values of the velocity and state variables are then interpreted as cell-centred averages. This is a fair approximation in the limit of moderate density jumps between fuel and ash. A caveat of using the passive implementation for simulations of burning at low density is the generation of numerical artifacts. Fortunately, these problems are mainly encountered for densities significantly less than about $10^{8} \mathrm{~g} \mathrm{~cm}^{-3}$. Apart from the systematic errors introduced by the averaged density and advection velocity, the burning zone is not strictly represented by the zero level set. Actually, there is a mixed phase between the regions containing pure ash and fuel, respectively. The width of the diffusive smearing of the flame is typically a few cells, which is still less than for the reaction-diffusion method. It was demonstrated by numerous applications in simple test problems as well as large-scale simulations of thermonuclear supernovae that the passive implementation gives a satisfactory representation of the flame fronts at high density and is robust even in three dimensions [34, 10]. For this reason, we used the passive implementation for the simulations presented in this article.

\subsection{The turbulent flame speed relation}

On length scales larger than the Gibson length $l_{\mathrm{G}}$, flames are predominantly shaped by turbulence. If $l_{\mathrm{G}}$ is significantly smaller than the numerical resolution $\Delta$, the computed flame front appears inevitably smoother than its physical counterpart. Consequently, the predicted burning rate would be un- 
derestimated, if just the laminar burning speed was substituted for the intrinsic propagation speed $s$ in equation (6). This is where the notion of the turbulent flame speed comes in. We propose that $s_{\mathrm{t}}(\Delta)$ is given by the local magnitude of unresolved turbulence velocity fluctuations $v^{\prime}(\Delta)$ [9], albeit the turbulent flame speed, in a strict sense, is an ensemble average. Since the propagation speed cannot be less than the laminar flame speed, the simplest relation with correct asymptotic behaviour is

$$
s_{\mathrm{t}}(\Delta)=\max \left(s_{\mathrm{lam}}, \sqrt{2 C_{\mathrm{t}} k_{\mathrm{sgs}}}\right)=\max \left(s_{\mathrm{lam}}, \sqrt{C_{\mathrm{t}}} q_{\mathrm{sgs}}\right)
$$

The quantity $k_{\mathrm{sgs}}=\frac{1}{2} q_{\mathrm{sgs}}^{2}$ is the subgrid scale turbulence energy and $q_{\mathrm{sgs}} \sim$ $v^{\prime}(\Delta)$ the corresponding speed. An exact definition will be given in next section. For brevity, it is understood that $s_{\mathrm{t}}$ denotes the turbulent flame speed at the numerical cutoff $\Delta$ in the following.

For $\Delta \gg l_{\mathrm{G}}$, we have the asymptotic relation $s_{\mathrm{t}} \approx \sqrt{C_{\mathrm{t}}} q_{\mathrm{sgs}}$ in the limit of fully developed turbulence. Consequently, the turbulent flame speed becomes independent of the laminar flame speed, and the parameter $\sqrt{C_{\mathrm{t}}}$ determines the asymptotic scaling of the turbulent flame speed. However, it is not quite clear whether the constant of proportionality in the relation between $s_{\mathrm{t}}(\Delta)$ and $q_{\mathrm{sgs}}$ is just unity or a different value. Empirically, it appears that $s_{\mathrm{t}}(\Delta)=2 v^{\prime}(\Delta)$, where $v^{\prime}(\Delta)=q_{\mathrm{sgs}} / \sqrt{3}$ [29. Thus, $C_{\mathrm{t}}=4 / 3$ in agreement with a constant of proportionality close to unity. Another shortcoming of the maximum relation (8) is that it gives a good approximation to the turbulent flame speed in the laminar and the fully turbulent regime, respectively, but not for the transition in between. If $q_{\mathrm{sgs}} \sim s_{\text {lam }}$, the relation between turbulent flame speed and turbulence velocity might very well be different. For example, Im et al. mention a quadratic dependence on the turbulence velocity in the case of weak turbulence [35. On the other hand, Röpke et al. report a linear relation even for turbulence velocities which are only marginally larger than the laminar flame speed in two-dimensional numerical simulations 36. However, this result is possibly unsubstantial for the three-dimensional case. Apart from that, the transition from laminar to turbulent burning progresses rather quickly, and a correct description in the intermediate phase is therefore not overly important.

A different turbulent flame speed model was motivated theoretically by Pocheau [37:

$$
\frac{s_{\mathrm{t}}}{s_{\text {lam }}}=\left[1+C_{\mathrm{t}}\left(\frac{q_{\mathrm{sgs}}}{s_{\text {lam }}}\right)^{n}\right]^{1 / n} .
$$

In the scale-invariant regime, with $q_{\mathrm{sgs}} \gg s_{\mathrm{lam}}$, the asymptotic form $s_{\mathrm{t}} \simeq$ 
$C_{\mathrm{t}}^{1 / n} q_{\text {sgs }}$ is obtained. Kim et al., chose $n=2$ and $C_{\mathrm{t}} \approx 20 / 3$ for LES of gas turbine combustor flows [23]. This value was inferred from several laboratory experiments with hydrocarbon/air flames. However, the data points cover values of the turbulent flame speed of the same order as magnitude as the laminar burning speed only. For this reason, one must be careful with any extrapolation to the fully turbulent regime, in which $s_{\mathrm{t}} \gg s_{\text {lam }}$. If $q_{\mathrm{sgs}} \ll s_{\text {lam }}$, Taylor series expansion of the right-hand side of equation (9) yields the Calvin-Williams relation,

$$
\frac{s_{\mathrm{t}}}{s_{\mathrm{lam}}}=1+C_{\mathrm{t}}\left(\frac{q_{\mathrm{sgs}}}{s_{\mathrm{lam}}}\right)^{2}
$$

which is consistent with the numerical results of Im et al. 35].

Apart from calculating the turbulent flame speed, secondary SGS effects can be included in the dynamical equation (6) for the level set function [23, 29]. This was indeed numerically investigated by Im et al. 35. In particular, they suggested a procedure for the computation of $C_{\mathrm{t}}$ in the fashion of the localised closure for the production parameter $C_{\nu}$, which will be discussed in section 4.2 . Whether this is advisable in combination with the passive implementation, where numerical artifacts in the shape of the resolved level set might produce significant spurious contributions, is questionable. For this reason, it has not been attempted. In addition, there is a SGS transport term for the level set, which is of the form $\partial_{k}\left(\left\langle v_{k}^{\infty} G\right\rangle_{\text {eff }}-v_{k} G\right)$ and effectively introduces diffusion of the level set due to SGS turbulence. However, Kim et al. argued that the contributions arising thereof are not particularly important and, in fact, cannot be determined within the available framework of SGS modelling [23].

\section{The subgrid scale model}

For the determination of the turbulent flame speed according to equation (8) or (9), the kinetic energy $k_{\mathrm{sgs}}$ of unresolved vortices has to be computed. A dynamical equation for $k_{\mathrm{sgs}}$ is obtained through decomposition of the conservation law for kinetic energy. The procedure of decomposing is conceptually based on the notion of filtered quantities. In general, a filter is a convolution operator, which smoothes out fluctuations on spatial scales smaller than the characteristic length of the filter. If a certain numerical solution of the hydrodynamical equations is computed by means of a finite-volume scheme, say, the PPM, then one can associate this solution with the smoothed velocity field $\boldsymbol{v}(\boldsymbol{x}, t)$, which is obtained by mass-weighted or Favre filtering of the exact 
realisation of the flow:

$$
\boldsymbol{v}(\boldsymbol{x}, t)=\frac{\langle\stackrel{\infty}{\rho}(\boldsymbol{x}, t) \stackrel{\infty}{\boldsymbol{v}}(\boldsymbol{x}, t)\rangle_{\mathrm{eff}}}{\rho(\boldsymbol{x}, t)}
$$

where $\rho(\boldsymbol{x}, t)=\langle\stackrel{\infty}{\rho}(\boldsymbol{x}, t)\rangle_{\mathrm{eff}}$ is the smoothed mass density. The underlying hypothesis is that, if the physical flow $\underset{\boldsymbol{v}}{\boldsymbol{x}}(\boldsymbol{x}, t)$ were known, there exists a filter \langle\rangle$_{\text {eff }}$ with suitable properties such that the Favre-filtered velocity field $\boldsymbol{v}(\boldsymbol{x}, t)$ would reproduce the numerically computed velocity field. Corresponding to the smoothed and the fluctuating components of $\underset{\boldsymbol{v}}{\infty}(\boldsymbol{x}, t)$, respectively, one can distinguish the resolved part, $k_{\mathrm{res}}=\frac{1}{2}|\boldsymbol{v}|^{2}$, and the subgrid scale part $k_{\mathrm{sgs}}$ of the specific kinetic energy. In the following, a formal decomposition of the kinetic energy is devised and the dynamical equation for $k_{\mathrm{sgs}}$ is formulated. The non-linearity of the conservation laws necessitates closure relations for several terms in the decomposed equations. SGS closures and the calculation of associated parameters are discussed in the remainder of this section.

\subsection{The subgrid scale turbulence energy model}

In Germano's consistent decomposition, the SGS turbulence energy is simply defined by the difference between smoothed and resolved kinetic energy 22, 38. This decomposition is equivalent to setting $k_{\mathrm{sgs}}=-\frac{1}{2} \tau_{i i}$, where the SGS turbulence stress tensor $\tau_{i k}$ is defined by

$$
\tau_{i k} \equiv \tau\left(\stackrel{\infty}{v_{i}}, \stackrel{\infty}{v_{k}}\right)=-\left\langle\stackrel{\infty \infty \infty}{\rho} v_{i} v_{k}\right\rangle_{\mathrm{eff}}+\rho v_{i} v_{k}
$$

Hence, the SGS turbulence energy is given by

$$
k_{\mathrm{sgs}}=\frac{1}{2}\left[\frac{1}{\rho}\left\langle\stackrel{\infty}{\rho}|\boldsymbol{v}|^{2}\right\rangle_{\mathrm{eff}}-|\boldsymbol{v}|^{2}\right] .
$$

We prefer the Germano decomposition, because it yields the conceptually most transparent definition of the SGS turbulence energy and avoids formal difficulties associated with SGS closures. Deviations of the turbulent flame speed in alternative decompositions correspond to higher-order terms which result from secondary filtering of filtered quantities [22]. These contributions are likely to be insignificant within the intrinsic inaccuracy of the flame speed model

The SGS turbulence stress tensor $\tau_{i k}$ enhances the viscous dissipation given by $\sigma_{i k}$ in the equation of motion for the filtered velocity field:

$$
\rho \frac{\mathrm{D}}{\mathrm{D} t} v_{i}=-\frac{\partial P}{\partial x_{i}}+\rho f_{i}^{(\mathrm{s})}+\frac{\partial}{\partial x_{k}}\left(\sigma_{i k}+\tau_{i k}\right) .
$$


Here it is assumed that the specific stirring force $f_{i}^{(\mathrm{s})}$ injects energy on length scales which are large compared to the numerical resolution $\Delta$. The operator $\mathrm{D} / \mathrm{D} t$ is the Lagrangian derivative,

$$
\frac{\mathrm{D}}{\mathrm{D} t}=\frac{\partial}{\partial t}+\boldsymbol{v} \cdot \nabla
$$

The dynamical equations for kinetic energy in the Germano decomposition read

$$
\begin{gathered}
\rho \frac{\mathrm{D}}{\mathrm{D} t} k_{\mathrm{res}}=v_{i}\left[-\frac{\partial P}{\partial x_{i}}+\rho f_{i}^{(\mathrm{s})}+\frac{\partial}{\partial x_{k}} \tau_{i k}\right] \\
\rho \frac{\mathrm{D}}{\mathrm{D} t} k_{\mathrm{sgs}}-\mathfrak{D}_{\mathrm{sgs}}=\Sigma_{\mathrm{sgs}}-\rho\left(\lambda_{\mathrm{sgs}}+\epsilon_{\mathrm{sgs}}\right) .
\end{gathered}
$$

In the first equation, the rate of viscous dissipation of kinetic energy, $\boldsymbol{\nabla} \cdot(\boldsymbol{v} \cdot \boldsymbol{\sigma})$, is neglected under the assumption that the flow is virtually unaffected by microscopic viscosity on length scales greater than the grid resolution $\Delta$. This is a valid approximation for $\Delta \gg \eta_{\mathrm{K}}$, where $\eta_{\mathrm{K}}$ is the Kolmogorov scale of viscous dissipation. For the numerical simulation discussed later-on, $\Delta \sim 10^{3} \mathrm{~cm}$, whereas $\eta_{\mathrm{K}} \ll 1 \mathrm{~cm} \mathrm{[24]}$. The symbolic terms in equation (17) account for the diffusion, production and dissipation of SGS turbulence energy (see [15] for the exact definitions). Energy transfer from resolved toward subgrid scales is given by the rate of production $\Sigma_{\text {sgs }}$. The non-local transport term $\mathfrak{D}_{\text {sgs }}$ accounts for the redistribution of turbulence energy by subgrid scale velocity and pressure fluctuations. Furthermore, there are two contributions to the rate of dissipation: $\rho \epsilon_{\mathrm{sgs}}$ is caused by the viscosity of the fluid, while $\rho \lambda_{\mathrm{sgs}}$ is due to compression effects. In fact, all of the SGS dynamical terms are non-computable in terms of resolved quantities. This is a consequence of the non-linear structure of the hydrodynamical equations, which prohibits complete decomposition. In consequence, one must find heuristic approximations in terms of computable quantities, which are commonly known as SGS closures.

We apply the customary turbulent-viscosity hypothesis for the rate of production (section 10.1 in 39]), the gradient-diffusion hypothesis for turbulent transport (section 4.3 in [22]) and the dimensional closure for the rate of viscous dissipation (section 13.6.3 in [39]). Since pressure effects are small for deflagration in degenerate matter, a rather crude closure for $\lambda_{\text {sgs }}$ is utilised [40]. 
The final result is the following dynamical equation (section 3.1.4 in [15]):

$$
\begin{aligned}
& \frac{\mathrm{D}}{\mathrm{D} t} k_{\mathrm{sgs}}- \frac{1}{\rho} \nabla \cdot\left(\rho C_{\kappa} \Delta_{\mathrm{eff}} k_{\mathrm{sgs}}^{1 / 2} \nabla k_{\mathrm{sgs}}\right)= \\
& C_{\nu} \Delta_{\mathrm{eff}} k_{\mathrm{sgs}}^{1 / 2}\left|S^{*}\right|^{2}-\left(\frac{2}{3}+C_{\lambda}\right) k_{\mathrm{sgs}} d-C_{\epsilon} \frac{k_{\mathrm{sgs}}^{3 / 2}}{\Delta_{\mathrm{eff}}} .
\end{aligned}
$$

In particular, an expression analogous to the viscous dissipation term in the Navier-Stokes equations, $\sigma_{i k}=\rho \nu S_{i k}$ is substituted for the anisotropic part of $\tau_{i k}$. The rate of SGS turbulence production is then given by

$$
\Sigma_{\mathrm{sgs}}=\tau_{i k} S_{i k}=\rho\left(\nu_{\mathrm{sgs}}\left|S^{*}\right|-\frac{2}{3} k_{\mathrm{sgs}} d\right),
$$

where $\nu_{\mathrm{sgs}}=C_{\nu} \Delta_{\mathrm{eff}} k_{\mathrm{sgs}}^{1 / 2}$ is the SGS turbulence viscosity. The rate-of-strain tensor $S_{i k}$ is the symmetrised spatial derivative of the velocity field:

$$
S_{i k}=v_{(i, k)} \equiv \frac{1}{2}\left(\frac{\partial v_{i}}{\partial x_{k}}+\frac{\partial v_{k}}{\partial x_{i}}\right)
$$

The trace of this tensor yields the dilatation of the velocity field, $d=S_{i i}$. The scalar $\left|S^{*}\right|$ is formed by total contraction of the trace-free part of the rate-of-strain tensor,

$$
\left|S^{*}\right|=\sqrt{2 S_{i k}^{*} S_{i k}^{*}}=\sqrt{2\left(S_{i k} S_{i k}-\frac{1}{3} d^{2}\right)} .
$$

The length scale $\Delta_{\text {eff }}$ is an effective scale of the finite-volume scheme, namely, the PPM. The ratio $\beta=\Delta_{\text {eff }} / \Delta$ specifies the smoothing of the flow on the smallest resolved scales due to numerical dissipation. In [41, we propose a method of calculating $\beta$ from numerical realisations of isotropic turbulence. For moderately compressible flows, it appears that $\beta \approx 1.6$.

Alternatively, a dynamical equation for the turbulence velocity $q_{\mathrm{sgs}}=$ $\sqrt{2 k_{\text {sgs }}}$ can be formulated:

$\frac{\mathrm{D}}{\mathrm{D} t} q_{\mathrm{sgs}}-\frac{1}{\rho} \boldsymbol{\nabla} \cdot\left(\rho \ell_{\kappa} q_{\mathrm{sgs}} \nabla q_{\mathrm{sgs}}\right)-\ell_{\kappa}\left|\nabla q_{\mathrm{sgs}}\right|^{2}=\ell_{\nu}\left|S^{*}\right|^{2}-\left(\frac{1}{3}+\frac{C_{\lambda}}{2}\right) q_{\mathrm{sgs}} d-\frac{q_{\mathrm{sgs}}^{2}}{\ell_{\epsilon}}$.

The length scales introduced above are defined as follows:

$$
\ell_{\kappa}=C_{\kappa} \Delta_{\text {eff }} / \sqrt{2}, \quad \ell_{\nu}=C_{\nu} \Delta_{\text {eff }} / \sqrt{2}, \quad \ell_{\epsilon}=2 \sqrt{2} \Delta_{\text {eff }} / C_{\epsilon} .
$$


The equation for $q_{\mathrm{sgs}}$ can be evolved starting with the initial data $q_{\mathrm{sgs}}(\boldsymbol{x}, 0)=0$ for a fluid being initially at rest. Moreover, non-integer powers of $q_{\text {sgs }}$ do not occur, and the functional dependence on $q_{\mathrm{sgs}}$ is advantageous for the discretisation of the diffusion term. However, numerical errors may arise from the nonconservative form of equation (21). Since we apply the solution predominantly to estimate the turbulent flame speed, this caveat is not of much concern.

At this point, one is left with the problem of determining the closure parameters $C_{\kappa}, C_{\nu}, C_{\epsilon}$ and $C_{\lambda}$. For isotropic turbulence, approximate statistical values from analytic theories or numerical data can be found. We adopted the constant parameter $C_{\lambda}=-0.2$ [42], and the turbulent diffusion parameter $C_{\kappa}=0.36$ was estimated from inertial-subrange properties of flow realisations in numerical simulations of forced isotropic turbulence (section 3.2.4 in [15]).

A more sophisticated approach is the numerical in situ computation of SGS closure parameters from local structural properties of the flow. The underlying idea is that turbulence in the inertial subrange becomes asymptotically self-similar towards smaller length scales. In other words, mostly turbulent velocity fluctuations on the smallest numerically resolved length scales determine the local energy transfer towards unresolved scales. This idea initiated the development of so-called dynamical procedures for the computation of $C_{\nu}$. The result is a localised closure for the rate of energy transfer $\Sigma_{\text {sgs }}$ (section 4.3 in [22]). For the rate of dissipation, $\epsilon_{\mathrm{sgs}}$, localised closures have been suggested as well. Given the computational difficulties and conceptual shortcomings of these closures, we decided to apply a statistical method for calculating timedependent mean dissipation parameters in regions containing fuel, flames or ash, respectively. In the following section, we will explain the computational procedures for $C_{\nu}$ and $C_{\epsilon}$ in detail. A generalisation including dynamical procedures for $C_{\kappa}$ or $C_{\lambda}$ as well would be extremely involved and is likely to be infeasible in terms of computational costs. Fortunately, the most important contributions to SGS dynamics arise from the production and the dissipation terms.

\subsection{The semi-localised model}

In order to extract the small scale velocity fluctuations in a simulation, a test filter \langle\rangle$_{\mathrm{T}}$ is applied. This filter smooths the numerically computed flow over a characteristic length $\Delta_{\mathrm{T}}=\gamma_{\mathrm{T}} \Delta_{\text {eff }}$, where the factor $\gamma_{\mathrm{T}}>1$. It is then possible to compute the turbulence stress associated with the intermediate range of length scales $\Delta \lesssim l \lesssim \Delta_{\mathrm{T}}$ :

$$
\tau_{\mathrm{T}}\left(v_{i}, v_{k}\right)=-\left\langle\rho v_{i} v_{k}\right\rangle_{\mathrm{T}}+\frac{1}{\langle\rho\rangle_{\mathrm{T}}}\left\langle\rho v_{i}\right\rangle_{\mathrm{T}}\left\langle\rho v_{k}\right\rangle_{\mathrm{T}},
$$


Applying the eddy-viscosity closure to the trace-free part of $\tau_{\mathrm{T}}\left(v_{i}, v_{k}\right)$, we have

$$
\tau_{\mathrm{T}}^{*}\left(v_{i}, v_{k}\right) S_{i k}^{[\mathrm{T}]}=\rho_{\mathrm{T}} C_{\nu} \Delta_{\mathrm{T}} k_{\mathrm{T}}^{1 / 2}\left|S^{*[\mathrm{~T}]}\right|^{2}
$$

where $\rho_{\mathrm{T}}=\langle\rho\rangle_{\mathrm{T}}$ is the test-filtered mass density, and the rate of strain at the test filter level is defined by $S_{i k}^{[\mathrm{T}]}=\partial_{(i}\left\langle\rho v_{j}\right\rangle_{\mathrm{T}} / \rho_{\mathrm{T}}$. The expression $C_{\nu} \Delta_{\mathrm{T}} k_{\mathrm{T}}^{1 / 2}$ has the dimension of viscosity. The kinetic energy $k_{\mathrm{T}}$ is defined analogous to equation (18), with the test filter in place of the implicit filter and the numerically computed velocity $\boldsymbol{v}$ in place of $\stackrel{\infty}{\boldsymbol{v}}$.

Invoking the similarity hypothesis that $C_{\nu}$ is equal for the eddy-viscosity closure at the test filter level and for the unfiltered SGS turbulence stress, the anisotropic part of the rate of production in the localised SGS model is given by

$$
\frac{1}{\rho} \Sigma_{\mathrm{sgs}}+\frac{2}{3} k_{\mathrm{sgs}} d \stackrel{\circ}{=} \ell_{\nu} q_{\mathrm{sgs}}\left|S^{*}\right|^{2}=\frac{\tau_{\mathrm{T}}^{*}\left(v_{i}, v_{k}\right) S_{i k}^{[\mathrm{T}]}}{\gamma_{\mathrm{T}} \rho_{\mathrm{T}}} \frac{\left|S^{*}\right|^{2}}{\left|S^{*[\mathrm{~T}]}\right|^{2}} \sqrt{\frac{k_{\mathrm{sgs}}}{k_{\mathrm{T}}}} .
$$

The above closure is basically the result of adapting the Germano-Lilly dynamical procedure for the localised Smagorinsky model to the SGS turbulence energy model [43. As an important difference, however, the eddy viscosity closure is applied to $\tau_{\mathrm{T}}\left(v_{i}, v_{k}\right)$ rather than the total turbulence stress at the test filter level, $\tau_{\mathrm{T}}\left(\stackrel{\infty}{v_{i}}, \stackrel{\infty}{k}_{k}\right)$. The stress tensors are related by the Germano identity [38]:

$$
\tau_{\mathrm{T}}\left(\stackrel{\infty}{v_{i}}, \stackrel{\infty}{v_{k}}\right)=\left\langle\tau\left(\stackrel{\infty}{v_{i}}, \stackrel{\infty}{v_{k}}\right)\right\rangle_{\mathrm{T}}+\tau_{\mathrm{T}}\left(v_{i}, v_{k}\right)
$$

This modification of the dynamical procedure was proposed by Kim et al. 23. It is supported by results from the evaluation of velocity measurements in round jets 44 and was explicitly verified on data from simulations of compressible turbulence driven by stochastic stirring (section 3.2.2 in [15]).

A complication arises from $C_{\nu}$ becoming negative in some regions of a turbulent flow. This is commonly interpreted as backscattering, i.e. kinetic energy is locally transfered across the cutoff from smaller, unresolved vortices towards vortices of size larger than $\Delta$ (section 4.4 in [22]). Including the contributions from backscattering in numerical simulations introduces several difficulties [45]. Firstly, numerical instabilities might be induced, because backscattering amounts to negative diffusion. Secondly, the SGS turbulence stresses must be coupled to the resolved flow in order to consistently account for the conversion of SGS turbulence energy into resolved kinetic energy. This is exactly what one would do in conventional large eddy simulations. However, in 
combination with a dissipative finite-volume scheme such as the PPM, including the SGS stress terms in the momentum equation does not do much good. Inevitably, the kinetic energy produced by positive SGS stress (corresponding to negative SGS viscosity) would be injected into modes corresponding to wave numbers near the cutoff. These wave numbers, however, are severely affected by numerical dissipation [41, and the fluid motion produced by the inverse energy transfer would be rapidly damped out. Consequently, backscattering would effectively result in enhanced dissipation, i.e. conversion of subgrid scale turbulence energy into internal rather than resolved kinetic energy. For this reason, the outcome of suppressing backscattering will be investigated in section 5.3

Finding a dynamical procedure for the parameter of SGS dissipation, $C_{\epsilon}$, is yet more demanding. The difficulty of determining $C_{\epsilon}$ stems from the fact the rate of dissipation is mostly determined by the fluid dynamics on scales much smaller than the numerical resolution. Therefore, a localised similarity hypothesis is bound to fail. However, one can invoke a statistical argument. Considering a certain region of the flow, the average rate of dissipation in that region should be roughly balanced by the mean transfer from larger toward smaller scales, if the flow is nearly in statistical equilibrium. Even in developing flows, a time-dependent statistical value of $C_{\epsilon}$ can be calculated by means of energy conservation. The method is loosely based on the variational approach of [46], where the parameter of dissipation $C_{\epsilon}$ is determined by subtracting the test-filtered SGS turbulence energy equation (17) from the corresponding equation for the unresolved kinetic energy at the level of the test filter. Rather than computing $C_{\epsilon}$ locally, we will determine statistical values evolving in time from the spatially averaged energy equations. Upon averaging equation (17), one obtains

$$
\left\langle\rho \frac{\mathrm{D}}{\mathrm{D} t} k_{\mathrm{sgs}}\right\rangle=\left\langle\tau_{i k} S_{i k}\right\rangle-\left\langle\rho\left(\lambda_{\mathrm{sgs}}+\epsilon_{\mathrm{sgs}}\right)\right\rangle
$$

The diffusion term cancels out, because integrating the divergence of the diffusive flux over a domain with periodic BCs yields zero. Furthermore,

$$
\left\langle\rho \frac{\mathrm{D}}{\mathrm{D} t} k_{\mathrm{sgs}}\right\rangle=\left\langle\frac{\partial}{\partial t} \rho k_{\mathrm{sgs}}\right\rangle+\underbrace{\left\langle\frac{\partial}{\partial x_{i}} \rho v_{i} k_{\mathrm{sgs}}\right\rangle}_{=0}=\frac{\mathrm{d}}{\mathrm{d} t}\left\langle K_{\mathrm{sgs}}\right\rangle,
$$

i.e. there is vanishing net advection over the whole domain of the flow. The turbulence energy at the characteristic scale of the test filter is defined by

$$
-\frac{1}{2} \tau_{\mathrm{T}}\left(\stackrel{\infty}{v_{i}}, \stackrel{\infty}{v_{i}}\right)=-\frac{1}{2}\left\langle\tau_{i i}\right\rangle_{\mathrm{T}}+\frac{1}{2} \tau_{\mathrm{T}}\left(v_{i}, v_{i}\right)=\left\langle\rho k_{\mathrm{sgs}}\right\rangle_{\mathrm{T}}+\rho_{\mathrm{T}} k_{\mathrm{T}},
$$


and the corresponding averaged dynamical equation is

$$
\frac{\partial}{\partial t}\left\langle\rho K_{\mathrm{sgs}}+\rho_{\mathrm{T}} K_{\mathrm{T}}\right\rangle=\left\langle\tau_{\mathrm{T}}\left(\begin{array}{l}
\infty \\
v_{i}
\end{array}, v_{k}\right) S_{i k}^{[\mathrm{T}]}\right\rangle-\left\langle\rho\left(\lambda_{\mathrm{sgs}}+\epsilon_{\mathrm{sgs}}\right)+\rho_{\mathrm{T}}\left(\lambda_{\mathrm{T}}+\epsilon_{\mathrm{T}}\right)\right\rangle .
$$

Equations (27) and (29) in combination with the Germano identity (26) imply the following conservation law for the mean turbulence energy $\left\langle K_{\mathrm{T}}\right\rangle$ associated with the smallest resolved scales:

$$
\frac{\mathrm{d}}{\mathrm{d} t}\left\langle K_{\mathrm{T}}\right\rangle=\left\langle\tau_{\mathrm{T}}\left(v_{i}, v_{k}\right) S_{i k}^{[\mathrm{T}]}+\left\langle\tau_{i k}\right\rangle_{\mathrm{T}} S_{i k}^{[\mathrm{T}]}-\tau_{i k} S_{i k}\right\rangle-\left\langle\rho_{\mathrm{T}}\left(\lambda_{\mathrm{T}}+\epsilon_{\mathrm{T}}\right)\right\rangle .
$$

Substituting the turbulent-viscosity closures for the various production terms on the right-hand side, the above equation becomes

$$
\begin{aligned}
\frac{\mathrm{d}}{\mathrm{d} t}\left\langle K_{\mathrm{T}}\right\rangle \simeq & \underbrace{\left\langle\rho_{\mathrm{T}} C_{\nu} \Delta_{\mathrm{T}} \sqrt{k_{\mathrm{T}}}\left|S^{*[\mathrm{~T}]}\right|^{2}\right\rangle-\frac{2}{3}\left\langle K_{\mathrm{T}} d^{[\mathrm{T}]}\right\rangle}_{(\mathrm{I})}-\left\langle\rho_{\mathrm{T}} \lambda_{\mathrm{T}}\right\rangle+\left\langle\rho_{\mathrm{T}} \epsilon_{\mathrm{T}}\right\rangle \\
& +\underbrace{\left\langle\left\langle\rho \nu_{\mathrm{sgs}} S_{i k}^{*}\right\rangle_{\mathrm{T}} S_{i k}^{*[\mathrm{~T}]}-\rho \nu_{\mathrm{sgs}}\left|S^{*}\right|^{2}\right\rangle}_{\text {(II) }}-\frac{2}{3} \underbrace{\left\langle\left\langle K_{\mathrm{sgs}}\right\rangle_{\mathrm{T}} d^{[\mathrm{T}]}-K_{\mathrm{sgs}} d\right\rangle}_{\text {(III) }} .
\end{aligned}
$$

Analogous to the rate of strain at the test filter level, the divergence $d^{[\mathrm{T}]}$ is given by $d^{[\mathrm{T}]}=\partial_{i}\left\langle\rho v_{i}\right\rangle_{\mathrm{T}} / \rho_{\mathrm{T}}$. The most significant production term is (I) which measures the energy transfer across the test filter scale $\Delta_{\mathrm{T}}$. The two contributions in term (II), on the other hand, are both related to the energy transfer across $\Delta_{\text {eff }}$, where the first expression is calculated from the testfiltered and the second expression from the numerically resolved rate of strain, respectively. It appears reasonable to assume that the difference of these two expressions is marginal relative to (I) in the case of scaling ratios $\Delta_{\mathrm{T}} / \Delta_{\text {eff }}$ of the order unity. Furthermore, the evaluation of (II) is particularly costly due to several tensor components which have to be test-filtered. Thus, we neglect term (II). For similar reasons and because of the smallness of compressibility effects, we drop (III) as well. In conclusion, the rate of dissipation $\epsilon_{\mathrm{T}}$ is approximately given by

$$
\left\langle\rho_{\mathrm{T}} \epsilon_{\mathrm{T}}\right\rangle \simeq-\frac{\mathrm{d}}{\mathrm{d} t}\left\langle K_{\mathrm{T}}\right\rangle+\left\langle\rho_{\mathrm{T}} C_{\nu} \Delta_{\mathrm{T}} \sqrt{k_{\mathrm{T}}}\left|S^{*[\mathrm{~T}]}\right|^{2}\right\rangle-\frac{2}{3}\left\langle\rho_{\mathrm{T}}\left(k_{\mathrm{T}} d^{[\mathrm{T}]}+\lambda_{\mathrm{T}}\right)\right\rangle .
$$

The crucial step is to conjecture that the relation between the spatially averaged dissipation rate and turbulence energy is similar at the cutoff and 
the test filter level. This implies

$$
\left\langle\rho_{\mathrm{T}} \epsilon_{\mathrm{T}}\right\rangle \stackrel{\circ}{=} \frac{C_{\epsilon}}{\Delta_{\mathrm{T}}}\left\langle\rho_{\mathrm{T}}\left(\frac{\left\langle\rho k_{\mathrm{sgs}}\right\rangle_{\mathrm{T}}}{\rho_{\mathrm{T}}}+k_{\mathrm{T}}\right)^{3 / 2}-\gamma_{\mathrm{T}} \rho k_{\mathrm{sgs}}^{3 / 2}\right\rangle .
$$

Note that the expression in parentheses is the total turbulence energy at the test filter level. For the pressure-dilatation term $\lambda_{\mathrm{T}}$, we set

$$
\lambda_{\mathrm{T}} \stackrel{\circ}{=} C_{\lambda} k_{\mathrm{T}} d^{[\mathrm{T}]}
$$

which is analogous to the closure at the subgrid-scale level. The rate of SGS dissipation is therefore given by

$$
\begin{aligned}
\epsilon_{\mathrm{sgs}}= & -\gamma_{\mathrm{T}}\left\langle\rho_{\mathrm{T}}\left(\frac{\left\langle\rho k_{\mathrm{sgs}}\right\rangle_{\mathrm{T}}}{\rho_{\mathrm{T}}}+k_{\mathrm{T}}\right)^{3 / 2}-\gamma_{\mathrm{T}} \rho k_{\mathrm{sgs}}^{3 / 2}\right\rangle^{-1} \\
& \times\left[\frac{\mathrm{d}}{\mathrm{d} t}\left\langle K_{\mathrm{T}}\right\rangle-\left\langle C_{\nu} \rho_{\mathrm{T}} \Delta_{\mathrm{T}} \sqrt{k_{\mathrm{T}}}\left|S^{*[\mathrm{~T}]}\right|^{2}\right\rangle+\left(\frac{1}{3}+C_{\lambda}\right)\left\langle K_{\mathrm{T}} d^{[\mathrm{T}]}\right\rangle\right] k_{\mathrm{sgs}}^{3 / 2} .
\end{aligned}
$$

As opposed to the statistical values for the SGS parameters for steady isotropic turbulence, the above equation yields a spatially constant parameter evolving in time. This method of calculating $C_{\epsilon}$ in combination with the dynamical procedure for $C_{\nu}$ makes up the semi-localised SGSTE model. For the numerical implementation, two further modifications were added.

On account of the anisotropy in the vicinity of a flame front, it seems advisable to average over the principal topological subdomains, namely, the interior, the exterior and the interface. The latter is identified by marking all grid cells within a certain maximal distance to those cells in which the level set function $G$ swaps its sign. With this procedure, the functions $C_{\epsilon}^{(\mathrm{a})}(t), C_{\epsilon}^{(\mathrm{b})}(t)$ and $C_{\epsilon}^{(\mathrm{f})}(t)$ are obtained for the mean dissipation parameters in ash, the burning zone and fuel, respectively. In the early stage, burning regions might encompass only a small volume fraction with relatively high surface to volume ratio. Hence, the corresponding spatial averages in equation (35) will not be sufficiently well behaved at the beginning. Although, the dynamics is dominated by the fuel domain at this point, both the enumerator and dominator in equation (35) are smoothed in time via convolution with an exponential damping function in order to remove strong oscillations in the ash and flame regions. The characteristic time scale of smoothing is prescribed by the parameter $T_{\epsilon}$. An appropriate choice for the time scale $T_{\epsilon}$ has to be found a posteriori. Setting $T_{\epsilon} \approx 0.1 T$ appears to be a good choice in order to get well behaved 
functions $C_{\epsilon}^{(\mathrm{a})}(t), C_{\epsilon}^{(\mathrm{b})}(t)$ and $C_{\epsilon}^{(\mathrm{f})}(t)$, without overly damping dynamical variations (section 4.3.4 in [15]).

\section{Numerical Simulations}

The simplest case study one can think of is the evolution of flame fronts in spatially homogeneous turbulent flows. To that end, we implemented a stochastic driving mechanism for the production of turbulence in a cubic domain subject to periodic boundary conditions 1514. The notion of a stochastic force field is outlined below. The computational domain is divided into a lattice of subcubes of size $L=X / \alpha$, where $L$ is the characteristic wavelength of the stochastic driving force and $X$ is the domain size. In the centre of each subcube, thermonuclear burning is ignited in small spherical regions located at time $t=0$, when the stirring force begins to act on the fluid. We chose $\alpha=2$, giving eight subcubes in the computational domain. This pattern is infinitely repeated in space by virtue of the periodic boundary conditions. Gravity is negligible at the scales under consideration (section 2.3.3 in [15]). Consequently, there are no buoyancy effects and turbulence is only produced by stirring.

The crucial parameter for the evolution of the burning process is the ratio $\xi=s_{\text {lam }} / V$, i.e. the ratio of the laminar burning speed to the characteristic velocity of the turbulent flow. Assuming developed turbulence, one can apply the Kolmogorov scaling law and estimate the magnitude of turbulent velocity fluctuations at a separation of the order to the Gibson length:

$$
v^{\prime}\left(l_{\mathrm{G}}\right) \sim V\left(\frac{l_{\mathrm{G}}}{L}\right)^{1 / 3} .
$$

The integral length $L$ and characteristic velocity $V$ specify the largest turbulent vortices in the flow. Setting $v^{\prime}\left(l_{\mathrm{G}}\right)=s_{\text {lam }}$, the scaling law for the Gibson length becomes

$$
l_{\mathrm{G}} \sim L\left(\frac{s_{\text {lam }}}{V}\right)^{3}=L \xi^{3}
$$

Obviously, $l_{\mathrm{G}}$ is very sensitive to value of $\xi$. Possible choices of $V$ are restricted by the speed of sound $c_{\mathrm{s}}$. Both $c_{\mathrm{s}}$ and $s_{\text {lam }}$ are mostly determined by the mass density, and so is the ratio $l_{\mathrm{G}} / L$. In the following, we will consider two distinct cases. For $\xi>0.1$ and sufficiently high resolution, the Gibson scale is just within the range of numerically resolved length scales. In this case, no SGS model is required for the flame dynamics and the propagation speed is more or less given by the laminar flame speed. If $\xi \ll 0.1$, on the other hand, 
it is impossible to resolve the flame completely. Then the burning process will enter a turbulent regime, in which the flame propagation speed is asymptotically given the SGS turbulence velocity $q_{\mathrm{sgs}}$. Prior to the discussion of the numerical simulations, we give a brief description of the stirring mechanism for the production of isotropic turbulent flow.

\section{$5.1 \quad$ Stochastic forcing}

The specific driving force $\boldsymbol{f}(\boldsymbol{x}, t)$ is composed in spectral space, using a threedimensional generalisation of the scalar Ornstein-Uhlenbeck process, as proposed in [14. The evolution of the Fourier transform $\hat{\boldsymbol{f}}(\boldsymbol{k}, t)$ is given by the following Langevin-type stochastic differential equation:

$$
\mathrm{d} \hat{\boldsymbol{f}}(\boldsymbol{k}, t)=-\hat{\boldsymbol{f}}(\boldsymbol{k}, t) \frac{\mathrm{d} t}{T}+F_{0} \sum_{j l m}\left(\frac{2 \sigma^{2}(\boldsymbol{k})}{T}\right)^{1 / 2} \delta\left(\boldsymbol{k}-\boldsymbol{k}_{j l m}\right) \mathbf{P}_{\zeta}(\boldsymbol{k}) \cdot \mathrm{d} \mathcal{W}_{t}
$$

The second term on the right hand side accounts for a random diffusion process, which is constructed from a three-component Wiener process $\mathcal{W}_{t}$. The distribution of each component is normal with zero mean and variance $\mathrm{d} t$. The wave vectors $\boldsymbol{k}_{j l m}$ are dual to the position vectors of the cells in the numerical discretisation of the fundamental domain. The symmetric tensor $\mathbf{P}_{\zeta}(\boldsymbol{k})$ is defined by the linear combination of the projection operators perpendicular and parallel to the wave vector. The components of $\mathbf{P}_{\zeta}(\boldsymbol{k})$ can be expressed as

$$
\left(\mathrm{P}_{i j}\right)_{\zeta}(\boldsymbol{k})=\zeta \mathrm{P}_{i j}^{\perp}(\boldsymbol{k})+(1-\zeta) \mathrm{P}_{i j}^{\|}(\boldsymbol{k})=\zeta \delta_{i j}+(1-2 \zeta) \frac{k_{i} k_{j}}{k^{2}}
$$

where the spectral weight $\zeta$ determines whether the resulting force field in physical space is purely solenoidal, dilatational or a combination of both. The variance $\sigma^{2}(\boldsymbol{k})$ specifies the spectrum of the force field. We use a quadratic function, which confines the modes of the force to a narrow interval of wavenumbers, $k \in\left[0,2 k_{0}\right]$. The wave number $k_{0}$ determines the integral length scale of the flow, $L=2 \pi / k_{0}$.

The root mean square of the specific driving force is determined by the characteristic magnitude $F_{0}$ and the weight $\zeta$ :

$$
f_{\mathrm{rms}}=\sum_{j l m}\left\langle\hat{\boldsymbol{f}}_{j l m}(t) \cdot \hat{\boldsymbol{f}}_{j l m}(t)\right\rangle \simeq\left(1-2 \zeta+3 \zeta^{2}\right) F_{0}^{2}
$$

Since $F_{0}$ has the physical dimension of acceleration, it can be expressed as the characteristic velocity $V$ of the flow divided by the integral time scale $T$, 
which is given by the auto-correlation time $T$ of the driving force (38). Setting $T=L / V$, we have $F_{0}=V / T=L V^{2}$, and, starting with a homogeneous fluid at rest, the flow is developing towards a fully turbulent steady state within about two integral time scales.

\subsection{Quasi-laminar burning}

To begin with, we shall consider the case $\xi \sim 1$. Then the laminar flame propagation is fast enough to burn the smallest numerically resolved eddies in less than a turn-over time. Consequently, subgrid scale turbulence does not affect the flame dynamics. An estimate of the characteristic velocity $V$ for given numerical resolution and laminar burning speed is readily obtained from relation (37). The effective range of length scales which can be resolved is roughly given by $L / \Delta_{\text {eff }}=N / \alpha \beta$, where $N=\Delta / X$ is the number of numerical cells in one dimension. For $l_{\mathrm{G}} \approx \Delta_{\text {eff }}$, we therfore must have

$$
V \approx\left(\frac{N}{\alpha \beta}\right)^{1 / 3} s_{\text {lam }} .
$$

For the simulation, which will be discussed in the following, we used $432^{3}$ grid cells. Setting $N=432$, relation (41) implies that at most $V \approx 4 s_{\text {lam }}$ is admissible. Given a moderate mass density, this would entail an extremely low Mach number. However, computing an almost incompressible flow with the PPM would be infeasible. On the other hand, for an initial density $\rho_{0} \approx$ $2.90 \cdot 10^{9} \mathrm{~g} \mathrm{~cm}^{-3}$, one obtains $s_{\text {lam }} \approx 1.05 \cdot 10^{7} \mathrm{~cm} \mathrm{~s}^{-1}$ through interpolation of numerical data taken from Timmes and Woosley [6]. The speed of sound for this density is $c_{0} \approx 9.70 \cdot 10^{8} \mathrm{~cm} \mathrm{~s}^{-1}$. Choosing $V=4 s_{\text {lam }} \approx 4.20 \cdot 10^{7} \mathrm{~cm} \mathrm{~s}^{-1}$, the characteristic Mach number is $V / c_{0} \approx 0.043$. This is quite small, but still computationally manageable with a fully compressible hydro code. The resulting Gibson length, $l_{\mathrm{G}} \approx 3.3 \Delta$, allows for some margin between $l_{\mathrm{G}}$ and $\Delta_{\text {eff }} \approx 1.6 \Delta$.

Actually, Landau-Darrieus instabilities would induce a small-scale cellular flame structure [47. Due to the significant numerical dissipation at length scales $l \lesssim 10 \Delta$ [41, the cellular structure will inevitably get smeared out if $l_{\mathrm{G}} \sim \Delta$. For this reason, an effective cellular propagation speed $s_{\text {cell }}$ slightly larger than $s_{\text {lam }}$ would be the correct intrinsic propagation speed in place of the laminar burning speed [24]. However, this effect is ignored, as the change of the Gibson scale due to the difference between $s_{\text {lam }}$ and $s_{\text {cell }}$ is only about a factor of two. Consequently, using $s_{\text {cell }}$ as intrinsic propagation speed would not change the flame dynamics dramatically.

Regarding the numerical distortion introduced by the passive implementa- 
tion, one should be on the safe side for mass densities larger than $10^{8} \mathrm{~g} \mathrm{~cm}^{-3}$. Apart from that, the randomisation caused by turbulence tends to diffuse any numerical artifacts. In this respect, propagating symmetric flame fronts, say, nearly planar or spherical flames, is a more demanding task. Furthermore, flow maps prepared form the simulation data clearly show a tight correlation between the shape of the front and the flow structure (see figure 31). If there were significant spurious propagation or deformation, the evolution of the front should become increasingly uncorrelated to the flow. In conclusion, the simulations which will be discussed subsequently are likely to give a sound description of the flame dynamics, albeit the shortcomings of the level set method in the passive implementation.

The progression of the deflagration in the course of the simulation is illustrated by the sequences of contour plots for the specific internal energy, the mass density and the rate-of-strain scalar in figures 1, 2] and 3 respectively. The contours of the zero level set are visible as thin white lines which separate dark regions containing unburned matter of low energy density from the brightly coloured regions containing processed material of high energy density. In the course of the first integral time $T$, the regions of burned material are expanding gradually. At the same time, they are stretched an folded by the solenoidal large-scale flow. In the following second integral time, vortices are generated on small scales. Subsequent to the peak of $\langle B\rangle$ at $\tilde{t}=t / T \approx 1.35$, ash encloses fuel rather than the other way around, and the density of the enclosed fuel is noticeably larger than the average density. Around $\tilde{t} \approx 1.5$ most of the fuel has already been consumed by the burning process, and the last fuel patches are disappearing quickly. Thus, the peak of burning is reached before turbulence is fully developed and the front propagation is affected only little by small-scale velocity fluctuations in this simulation. Consequently, we refer to this mode of burning as quasi-laminar.

In panel (b) of figure 4, the mean burning rate $\langle B\rangle$ is plotted on a dimensionless scale. One can see that the rate of burning increases exponentially in the interval $0.3 \lesssim \tilde{t} \lesssim 1.2$. The norm $\left|S^{*}\right|$ of the trace-free rate of strain defined in equation (20) is a so-called structural invariant of the flow. Contour plots of $\left|S^{*}\right|$ are shown in figure 3. Regions which are subject to intense strain correspond to steep velocity gradients and appear bright in the contour plots. These regions tend to form vortices and clearly influence the morphology of the flame fronts. As one can see in figure 3, the white lines indicating the zero level set tend to be aligned with structures associated with large strain. The statistics of $\left|S^{*}\right|$ and two further structural invariants, namely, the vorticity $\omega=|\boldsymbol{\nabla} \times \boldsymbol{v}|$ and the divergence $d=\boldsymbol{\nabla} \cdot \boldsymbol{v}$, is plotted in panel (d) of figure 4 Because of the small characteristic Mach number of the flow, we have $d \ll \omega \simeq\left|S^{*}\right|$, where the equality of vorticity and rate-of-strain scalar holds asymptotically in the limit of incompressible flow. The graphs show that the 
dns_energy_evol.png

Figure 1. Three-dimensional simulation of thermonuclear deflagration in a cube with the flame propagation speed being equal to the laminar burning speed. The initial density of the $\mathrm{C}+\mathrm{O}$ fuel is $\rho_{0} \approx 2.90 \cdot 10^{9} \mathrm{~g} \mathrm{~cm}^{-3}$, and $V=4 s_{\text {lam }}(\xi=0.25)$. The characteristic Mach number of the fully developed turbulent flow is $V / c_{0} \approx 0.043$, where $c_{0}$ is the initial sound speed. Shown are two-dimensional contour sections of the normalised specific energy $\tilde{e}=e / c_{0}^{2}$ at different stages of the burning process.

root mean square (RMS) of $\left|S^{*}\right|$, grows exponentially from the first few tenths of an integral time scale up to $t / T \approx 2$, where the stagnation of the growth marks fully developed turbulence. A comparison between panels (b) and (d) suggests that the growth of the burning rate prior to the maximum correlates with the exponentially increasing $\left\langle\left|S^{*}\right|^{2}\right\rangle^{1 / 2}$. This underlines the above statement about the influence of strain onto the flame evolution. The corresponding evolution of the RMS momentum and Mach number is plotted in panel (a).

The rate of change of the mean mass fraction of fuel, $\langle\dot{X}(\mathrm{C}+\mathrm{O})\rangle$, is also a measure of the burning speed. For an energy release $\epsilon_{\text {nuc }}$ per unit mass, the total nuclear energy generated in the whole cubic domain per unit time can 


\section{dns_densty_evol.png}

Figure 2. 2D contour sections of the relative density fluctuations $\left(\rho-\rho_{0}\right) / \rho_{0}$ corresponding to the panels shown in figure 1

be expressed as

$$
(\alpha L)^{3}\langle B\rangle=-\epsilon_{\mathrm{nuc}} M_{\mathrm{cub}}\langle\dot{X}(\mathrm{C}+\mathrm{O})\rangle
$$

where $M_{\text {cub }}=\rho_{0}(\alpha L)^{3}$ is the total mass contained in the computational domain. On the other hand, the rate of energy production is related to the total surface area of the flames, $A_{\mathrm{F}}$, and the laminar propagation speed, provided that compression effects are neglected:

$$
(\alpha L)^{3}\langle B\rangle=\rho_{0} \epsilon_{\mathrm{nuc}} A_{\mathrm{F}} s_{\mathrm{lam}}
$$

Combining equations (42) and (43) with $M_{\text {cub }}=8 \rho_{0} L^{3}$, the approximate total 
Figure 3. 2D contour sections of the logarithmic dimensionless rate of strain, $\log _{10}\left(T\left|S^{*}\right|\right)$, corresponding to the panels shown in figures 1 and 2

surface area is given by

$$
A_{\mathrm{F}} \simeq \frac{(2 L)^{3}}{s_{\text {lam }}}\langle\dot{X}(\mathrm{C}+\mathrm{O})\rangle .
$$

The graph of the normalised surface area,

$$
\tilde{A}_{\mathrm{F}}=\frac{A_{\mathrm{F}}}{8 \pi^{2} L^{2}}=-\frac{1}{\pi^{2}} \frac{V}{s_{\text {lam }}}\langle T \dot{X}(\mathrm{C}+\mathrm{O})\rangle
$$

is shown in the panel (c) of figure 4 The exponential growth of the burning rate is manifest in this plot as well. At the peak, $\tilde{A}_{\mathrm{F}} \sim 1$, which verifies that the flames experience only little wrinkling due to small vortices. This result 
dns_stat3d.png

Figure 4. Evolution of dimensionless statistical moments for the simulation illustrated in the series of figures 10 2 and 3 The top panels show plots of the RMS force, momentum and Mach number as well as the average nuclear energy generation rate in combination with the chemical composition. Furthermore, a measure for the mean flame speed and the averaged structural invariants of the flow are plotted in the panels at the bottom.

agrees with the impression of rather smooth flames in figure 1 Also plotted is the graph of $-\langle T \dot{X}(\mathrm{C}+\mathrm{O})\rangle /\langle X(\mathrm{C}+\mathrm{O})\rangle$, which is a measure of the ratio of the flame surface area to the amount of still unburned material. ${ }^{1}$ The mean of this ratio follows a nearly exponential law even beyond the peak of $\langle B\rangle$ and, thus, can be considered as an invariant measure for the burning intensity even when the flames are already diminishing.

${ }^{1}$ Strictly, the volume of fuel left at a certain time would be given by $(\alpha L)^{3}\langle\rho X(\mathrm{C}+\mathrm{O})\rangle / \rho_{0}$. However, the mass-weighted fraction of $\mathrm{C}+\mathrm{O}$ was not calculated in the simulation. 


\subsection{Burning dominated by turbulence}

In the case $\xi \ll 1$, i.e. the laminar burning speed is small compared to the chacteristic velocity of the flow, the range of length scales between the Gibson scale and the integral length scale becomes very large. Consequently, it is impossible to resolve the flame dynamics completely. For example, setting $\rho_{0} \approx 2.90 \cdot 10^{8} \mathrm{~g} \mathrm{~cm}^{-3}$, which is by an order of a magnitude smaller than the density chosen in section 5.2 yields $s_{\text {lam }} \approx 9.78 \cdot 10^{5} \mathrm{~cm} \mathrm{~s}^{-1}$. Choosing a characteristic velocity $V=100 s_{\text {lam }}$, we have the Mach number $V / c_{0} \approx 0.15$, and the Gibson scale becomes $l_{\mathrm{G}} \sim 10^{-6} L$. Obviously, $l_{\mathrm{G}} \ll \Delta$ for any feasible numerical resolution. A subgrid scale model is therefore mandatory. In this section, several simulations of thermonuclear deflagration in the cube with the turbulent flame speed given by equations (8) and (9), respectively, are discussed. The SGS turbulence velocity $q_{\mathrm{sgs}}$ is computed via equation (21). The initial mass density and the characteristic velocity are as specified above. Otherwise, the same parameters as in section 5.2 are used. However, the resolution is reduced by a factor two. So there are $216^{3}$ grid cells.

A couple of simulations were performed with the semi-localised model and the maximum relation (8) with $C_{\mathrm{t}}=1$. In one case, we coupled the SGS stresses to the resolved flow and included backscattering, whereas no coupling was applied and backscattering was suppressed by setting the SGS viscosity parameter equal to $C_{\nu}^{+}=\max \left(0, C_{\nu}\right)$ in the other case. The validity of neglecting the SGS stress terms in the momentum equation (14) has been investigated in several hydrodynamical simulations with PPM [4, 41]. For combustion problems, the SGS model then runs in a passive mode and provides the turbulent flame speed only.

If negative values of $C_{\nu}$ are admissible, however, the SGS stress terms must be included, because otherwise backscattering would convert SGS turbulence energy into heat rather than kinetic energy on resolved scales. Hence, backscattering necessitates an active SGS model. For a fully consistent treatment, the terms $\boldsymbol{v} \cdot(\boldsymbol{\nabla} \cdot \boldsymbol{\tau})$ and $\rho \epsilon_{\mathrm{sgs}}$ have to be added on the right hand side of the conservation law for the total energy $e_{\text {tot }}=\frac{1}{2}|\boldsymbol{v}|^{2}+e_{\text {int }}$, which account for the transfer of kinetic energy between resolved and subgrid scales and the production of internal energy due to the viscous dissipation of SGS turbulence energy, respectively.

On the other hand, if backscattering is suppressed, a considerably simplified scheme is applied, where the dissipation of kinetic energy is solely of numerical origin, and $q_{\mathrm{sgs}}$ is treated as a passive scalar. In order to account for the exchange of energy between the resolved total energy, $e_{\text {tot }}=\frac{1}{2} v^{2}+e_{\text {int }}$, and the SGS turbulence energy in a rudimentary fashion, the Lagrangian rate of change of $k_{\mathrm{sgs}}$ is subtracted from the the conservation law for the total energy. Locally, this introduces a certain error due to the diffusive transport of SGS 
turbulence energy. In fact, the transport term in equation (18) changes the unresolved energy without affecting the resolved energy budget. We ignore this contribution in the energy update, if backscattering is suppressed and the SGS model is not completely coupled to the resolved flow. In the case of complete coupling, however, the exact SGS transfer and dissipation rate are accounted for in the dynamical equation for $e_{\text {int }}$. Of course, complete coupling would seem appropriate regardless of the treatment of inverse energy transfer. However, if backscattering is suppressed, we found that abandoning the SGS stresses and using the approximate energy update as outlined above changes the results only little, while increasing the computational efficiency significantly.

The test filter for the computation of the production parameter in the semilocalised SGS model is numerically implemented as follows. Orthogonal onedimensional mesh filters with nine supporting nodes are applied in the direction of each coordinate axis. The filter weights are determined by matching the Fourier transform of the kernel as accurately as possible to the spectral representation of an analytic box filter. Then a single free parameters remains, which is the filter scaling ratio $\gamma_{\mathrm{T}}$. For a given number of supporting nodes, $N_{\mathrm{T}}$, an optimal value of $\gamma_{\mathrm{T}}$ can be found from further constraining the Fourier transforms of the mesh and the analytic box filter, respectively, to be equal at the cutoff wavelength (appendix A.1.1 in [15]). In the case $N_{\mathrm{T}}=9$, we have $\Delta_{T} \approx 3.75 \Delta_{\text {eff }} \approx 6.74 \Delta$. Although $\gamma_{\mathrm{T}} \approx 3.75$ is considerably larger than a factor of two, which is commonly suggested in the literature, we obtained optimal results with this setting rather than with test filters of smaller characteristic length (section 4.3.3 in [15]).

Statistical results from the simulations are shown in figures 5 and [6. The evolution of the mean burning rate $\langle B\rangle$ and the corresponding fuel consumption together with the helium and nickel production is shown in the bottom panels $(\mathrm{g}, \mathrm{h}, \mathrm{i})$ of figure 5 . For comparison, the RMS forcing, momentum and Mach number are plotted in top row of panels $(a, b, c)$ for each simulation. In the course of the first integral time, the slope of $\langle B\rangle$ in logarithmic scaling is rather slowly rising. This indicates predominantly laminar burning. The oscillations of $\langle B\rangle$ at early time are caused by numerical discretisation errors, since the burning regions initially tend to become elongated into thin shapes and, in consequence, are only marginally resolved. As the flow becomes increasingly turbulent and a growing fraction of the total flame surface is subject to an enhanced propagation speed $s_{\mathrm{t}} \gg s_{\mathrm{lam}}$, the rate of burning rises rapidly. Eventually, the phase of exponentially growing energy release passes over into fading combustion once the greater part of the fuel has been exhausted. The transition point between the quasi-laminar and the turbulent burning phase can be estimated from the tangents to the almost linear portions of the graph of $\langle B\rangle$ in logarithmic scaling. By means of the plot of the mass-weighted statistical moments of $q_{\mathrm{sgs}}$ in the panels (d,e,f), one can see that the transition 
Figure 5. Evolution of dimensionless statistical moments for simulations with different flame speed models and SGS closures. The normalised RMS of the specific stirring force and the resolved momentum of the flow are shown in the top panels. The first three moments of the SGS turbulence velocity are plotted in the middle row of panels. Note that the mass-weighted mean of $q_{\text {sgs }}$ is scaled in units of the laminar burning speed $s_{\text {lam }}$. In the bottom panels, the mean burning rate and the average mass fractions of fuel and processed nuclei are shown.

coincides with $\left\langle\rho q_{\mathrm{sgs}}\right\rangle /\left(\rho_{0} s_{\text {lam }}\right) \approx 3$. The somewhat larger threshold value of the mean SGS turbulence velocity relative to the laminar burning speed for the onset of rapid turbulent burning is possibly a consequence of intermittency. This is also indicated by the large standard deviation, $\sigma\left(\rho q_{\mathrm{sgs}}\right)$, which is comparable to the mean, $\left\langle\rho q_{\mathrm{sgs}}\right\rangle$, during the production phase. The massweighted skewness, skew $\left(\rho q_{\mathrm{sgs}}\right)$, is particularly large in the early phase when eddies are forming locally, whereas it approaches a value near unity in the regime of statistically stationary and homogeneous turbulence.

Comparing the left and the middle column of plots in figure 5, the evolution of the burning process in the simulations which differ only by the coupling of 
Figure 6. Comparison between two different variants of the semi-localised SGS model. Backscattering is included in one case, whereas it is suppressed in the other case. The top panels show plots of the mean production parameter $C_{\nu}$ in regions containing ash, flames and fuel, respectively. The middle panels show the corresponding plots of the dissipation parameter, and the different contributions in equation [21] for the time evolution of $q_{\mathrm{sgs}}$ are plotted in the bottom panels.

the SGS model to the momentum equation and the treatment of inverse energy transfer appear quite similar. This can be seen in figures 7 and 9 as well, which show contour plots of the total energy per unit mass at different instants of time for both simulations. However, the burning process proceeds faster in the simulation without backscattering in comparison to the simulation with the fully coupled SGS model. Correspondingly, the peak of $\langle B\rangle$ is delayed in the latter case.

The differences in the SGS model are illustrated in figure 6. The averages of the production parameter $C_{\nu}$ in regions containing, respectively, ash, flames and fuel are plotted in panels (a) and (b), respectively. If backscattering is 
Figure 7. Simulation of thermonuclear deflagration for an initial density $\rho_{0} \approx 2.90 \cdot 10^{8} \mathrm{~g} \mathrm{~cm}^{-3}$. $V / s_{\text {lam }}=100$, corresponding to a characteristic Mach number $V / c_{0} \approx 0.15$. The turbulent flame speed is given by $s_{\mathrm{t}}=\max \left(s_{\text {lam }}, q_{\mathrm{sgs}}\right)$. The turbulent velocity $q_{\mathrm{sgs}}$ is computed with the semi-localised SGS model, where $C_{\nu}^{+}=\max \left(0, C_{\nu}\right)$ is set as parameter of turbulence production. Shown are $2 \mathrm{D}$ contour sections of the normalised specific energy $\tilde{e}=e / c_{0}^{2}$ at different stages of the burning process.

included, the mean of $C_{\nu}$ within the fuel is initially small. In the course of turbulence production, the parameter is growing and, eventually, $\left\langle C_{\nu}\right\rangle \approx 0.04$ in the statistically stationary regime. On the other hand, if backscattering is suppressed, the mean of $C_{\nu}$ changes only little in the dominating topological region. The local values of $C_{\nu}$ are fluctuating considerably for $\tilde{t}<1$. This can be seen from the strongly oscillating averages of $C_{\nu}$ in ash and flames, which initially fill quite narrow spatial regions and encounter varying conditions while being advected by the flow. The time evolution of the dissipation parameters shown in panels (c) and (d), on the other hand, exhibits more or less the same trend. During the first turn-over time, $C_{\epsilon}$ vanishes identically. At time $\tilde{t} \approx 1$, 
les_nb_q_sgs_evol.png

Figure 8. 2D contour sections of SGS turbulence velocity relative to the laminar burning speed, $q_{\mathrm{sgs}} / s_{\text {lam }}$, in logarithmic scaling. The panels correspond to those shown in Figure 7

small turbulent vortices begin to form and turbulent dissipation sets in. In statistical equilibrium, $C_{\epsilon}$ assumes a nearly constant value of about 0.65 for the fully coupled model and 0.75 without backscattering. In the latter case, a larger dissipation rate compensates the suppressed inverse energy transfer, as one can see from the plots of the mean rate of production and dissipation corresponding to the source terms on the right hand side of equation (21) in panels (e) and (f) of figure 6. It is obvious that complete coupling significantly reduces the rate of turbulence production. Nevertheless, the mean value of $q_{\mathrm{sgs}}$ is found to be nearly the same in the statistically stationary regime for both simulations. As mentioned in section 4.2. we suspect that turbulence production is systematically underestimated by the fully coupled SGS model in combination with the PPM, because the kinetic energy injected through backscattering into modes of high wave number will be quickly dissipated by 
Figure 9. Simulation with the same parameter as specified in the caption of figure 7 however, with $C_{\nu}$ as production parameter and complete coupling of SGS stresses to the flow.

numerical viscosity. An impression of the spatiotemporal evolution of $q_{\mathrm{sgs}}$ is given by the contour plots in figures 8 and [10] respectively.

On account of results from laboratory measurements, Kim et al. argue that Pocheau's relation (9) with $n=2$ and $C_{\mathrm{t}}=20 / 3$ gives the most accurate prediction of the turbulent flame speed [23]. The outcome of running a simulation with the flame speed relation

$$
s_{\mathrm{t}}=s_{\mathrm{lam}} \sqrt{1+\frac{20}{3}\left(\frac{q_{\mathrm{sgs}}}{s_{\mathrm{lam}}}\right)^{2}},
$$

is demonstrated by the statistics in the colum of panels $(c, f, i)$ on the very right of figure 5 Backscattering is also suppressed in this simulation. Now the peak 
Figure 10. 2D contour sections of SGS turbulence velocity relative to the laminar burning speed, $q_{\mathrm{sgs}} / s_{\text {lam }}$ in logarithmic scaling. The panels correspond to those shown in Figure 9

of the burning rate is reached even faster than in the case of the simulation with $C_{\mathrm{t}}=1$. In fact, the bulk of the burning takes place when the level of SGS turbulence is quite low. Accordingly, the plots of contour sections of the specific energy in figure 11] show that the flame surface is smoother and less corrugated by the flow in the course of the burning process. The slope of $\langle B\rangle$ is steepening significantly just for $\left\langle\rho q_{\mathrm{sgs}}\right\rangle /\left(\rho_{0} s_{\text {lam }}\right) \approx 1$. This would suggest that $C_{\mathrm{t}}=20 / 3$ is, indeed, a feasible choice. On the other hand, $C_{\mathrm{t}}=4 / 3$ is favoured by Peters 29], which yields more or less the same behaviour as in the case $C_{\mathrm{t}}=1$. From our current understanding, we should consider $C_{\mathrm{t}}$ as a parameter of the flame speed model, which is to be chosen within reasonable limits and validated a posteriori by the results obtained for a particular application. 


\section{Conclusion}

The numerical simulation of thermonuclear deflagration in a box subject to stochastic stirring was utilised as a test problem for the study of flame speed models. The evolution of the flame front was computed by means of the level set method in the so-called passive implementation. Essentially, an effective flame propagation speed must be calculated, if the Gibson scale is small compared to the resolution of the computational grid. A subgrid scale (SGS) model based on the budget of turbulence energy determines a velocity scale which is proportional to the propagation speed of flame fronts in the fully turbulent regime. Some of the closure parameters of the SGS model are locally calculated 
with dynamical procedures. Thus, we have a semi-localised model.

Particularly, we compared two variants of this model. In one case, inverse energy transfer from subgrid toward resolved scales was included, in the other case it was suppressed. Inverse energy transfer is also known as backscattering. For a consistent treatment of backscattering, complete coupling of the SGS model and the resolved hydrodynamics is indispensable. In combination with the piece-wise parabolic method, this entails difficulties stemming from the significant numerical viscosity of the scheme. But we obtained sensible results when suppressing backscattering and applying a simplified SGS model with partial coupling.

Depending on the constant of proportionality $\sqrt{C_{\mathrm{t}}}$ in the asymptotic flame speed relation, we found the transition from laminar to turbulent burning at noticeably different points in the course of turbulence production. This transition comes about once the SGS turbulence velocity must exceed the laminar burning speed in a significant volume fraction of the computational domain. Then the nuclear energy generation grows at a much higher rate, and the flame surface develops an intricate structure due to the stretching and folding caused by turbulent vortices. If $C_{\mathrm{t}}$ is about unity, the peak of nuclear energy release appears roughly when the turbulent flow becomes statistically stationary and homogeneous. For larger values of $C_{\mathrm{t}}$, most of the fuel is consumed in advance of turbulence becoming fully developed. We propose to consider $C_{\mathrm{t}}$ as a control parameter, which regulates the overall rapidness of the burning process.

The semi-localised SGS model presented here is especially suitable for any kind of transient and inhomogeneous turbulent combustion process. It is the first implementation of this kind of SGS model for an astrophysical application, namely, the numerical simulation of thermonuclear supernovae.

\section{Acknowledgements}

The simulations were run on the Hitachi SR-8000 of the Leibniz Computing Centre the IBM p690 of the Computing Centre of the Max-Planck-Society in Garching, Germany. We thank M. Reinecke for his helpful remarks concerning the level set method. One of the authors (W Schmidt) was supported by the priority research program Analysis and Numerics for Conservation Laws of the Deutsche Forschungsgesellschaft. The research of W Schmidt and J C Niemeyer was supported by the Alfried Krupp Prize for Young University Teachers of the Alfried Krupp von Bohlen und Halbach Foundation. 


\section{References}

[1] Hoyle, F. and Fowler, W. A., 1960, Astrophys. J., 132, 565

[2] Shapiro, S. L. and Teukolsky S. A., 1983, Black Holes, White Dwarfs and Neutron Stars, John Wiley \& Sons

[3] Woosley, S. E., Wunsch, S. and Kuhlen, M., 2004, Astrophys. J., 607, 921-930

[4] Hillebrandt, W. and Niemeyer, J. C., 2000, Ann. Rev. Astron. Astrophys., 38, 191

[5] Nomoto, K., Thielemann, F. K. and Yokoi, K., 1984, Astrophys. J., 286, 644-658

[6] Timmes, F. X. and Woosley, S. E., 1992, Astrophys. J., 396, 649-667

[7] Khokhlov, A. M., 1995, Astrophys. J., 449, 695

[8] Bell, J. B., Day, M. S., Rendleman, C. A., Woosley, C. A. and Zingale, M., 2004, Astrophys. J., 608, 883-906

[9] Niemeyer, J. C., and Hillebrandt, W., 1995, Astrophys. J., 452, 769

[10] Reinecke, M., Hillebrandt, W. and Niemeyer, J. C., 2002, Astron. \& Astrophys., 391, 1167-1172

[11] Gamezo, V. N., Khokhlov, A. M., Oran, E. S., Chtchelkanova, A. Y. and Rosenberg, R. O., 2003, Science, 299, 77-81

[12] Khokhlov, A. M., 1991, Astron. ES Astrophys., 245, 114-28

[13] Gamezo, V. N., Khokhlov, A. M. and Oran, E. S., Phys. Rev. L., 92, 211102

[14] Eswaran, V. and Pope, S. B., 1988 Comput. Fluids, 16, 257-278

[15] Schmidt, W., 2004, Turbulent Thermonuclear Combustion in Degenerate Stars, PhD Thesis, Technical University of Munich. http://tumb1.biblio.tu-muenchen.de/publ/diss/ph/2004/schmidt.html

[16] Osher, S. and Sethian, J. A., 1988, J. Comp. Phys., 79, 12-49

[17] Reinecke, M., Hillebrandt, W., Niemeyer, J. C., Klein, R. and Gröbl, A., 1999, Astron. 8 Astrophys., 347, 724-733

[18] Steinmetz, M., Müller, E. and Hillebrandt, W., 1992, Astron. \& Astrophys., 254, 177-190

[19] Reinecke, M., 2001, Modeling and simulation of turbulent combustion in type Ia supernovae, PhD Thesis, Technical University of Munich. http://tumb1.biblio.tu-muenchen.de/publ/diss/ph/2001/reinecke.html

[20] Colella, P. and Woodward, P. R., 1984, J. Comp. Phys., 54, 174-201

[21] Niemeyer, J. C. and Kerstein, A. R., 1997, New Astron., 2, 239-44

[22] Sagaut, P., 2001, Large Eddy Simulation for Incompressible Flows, Springer

[23] Kim, W., Menon, S. and Mongia, H. C., 1999, Combust. Sci. and Tech., 143, 25-62

[24] Niemeyer, J. C. and Woosley, S. E., 1997, Astrophys. J., 475, 740-753

[25] Landau, L. D. and Lifshitz, E. M., 1991, Lehrbuch der theoretischen Physik VI. Hydrodynamik, Akademie Verlag

[26] Kolmogorov, A. N., 1941, C. R. Acad. Sci. URSS 30, 301

[27] Damköhler, G., 1940, Z. Elektrochem., 46, 601-626

28] Kerstein, A. R., 1988, Combustion Sci. Technol., 50, 441

[29] Peters, N., 1999, J. Fluid Mech., 384, 107-132

[30] Williams, F. A., 1994, Turbulent reacting flows, Academic Press

[31] Sethian, 1996, Level Set Methods, Cambridge University Press

[32] Röpke, F. K., Niemeyer, J. C. and Hillebrandt, W., 2003, Astrophys. J., 588, 952-961

[33] Schmidt, H. and Klein, R., 2003, Combust. Theory Modelling, 7, 243-267

[34] Reinecke, M., Hillebrandt, W. and Niemeyer, J. C., 1999 Astron. ES Astrophys., 347, 739-747

[35] Im, H. G., Lund, T. S. and Ferziger, J. H., 1997 Phys. Fluids, 9, 3826-3833

[36] Röpke, F. K., Hillebrandt, W. and Niemeyer, J. C., 2004, Astron. \& Astrophys., 421, 783-795

[37] Pocheau, A., 1994, Phys. Rev. E, 49, 1109-1122

[38] Germano, M., 1992, J. Fluid Mech., 238, 325-336

[39] Pope, S. B., 2000, Turbulent Flows, Cambridge University Press

[40] Deardorff, J. W., 1973, ASME J. Fluids Engng., 429-38

[41] Schmidt, W., Hillebrandt, W. and Niemeyer, J. C., 2005 accepted by Comp. Fluids (preprint astro-ph/0407616)

[42] Fureby, C., Tabor, G., Weller, H. G. and Gosman, A. D., 1997 Phys. Fluids, 9, 3578-3580

[43] Germano, M., Piomelli, U., Moin, P. and Cabot, W. H., 1991, Phys. Fluids, 3, 1760-1765

[44] Liu, S., Meneveau, C. and Katz, J., 1994, J. Fluid Mech., 275, 83-119

[45] Piomelli, U., 1993, Phys. Fluids A, 5, 1484-1490

[46] Ghosal, S., Lund, T. S., Moin, P. and Akselvoll, K., 1995, J. Fluid Mech., 286, 229-255

[47] Röpke, F. K., Hillebrandt, W. and Niemeyer, J. C., 2004, Astron. Es Astrophys., 420, 411-422

[48] Sytine, I. V., Porter, D. H., Woodward, P. R., Hodson, S. W., Winkler, K., 2000, Comp. Phys. 158, 225-238 
This figure "dns_densty_evol.png" is available in "png" format from: http://arxiv.org/ps/astro-ph/0508076v1 
This figure "dns_energy_evol.png" is available in "png" format from: http://arxiv.org/ps/astro-ph/0508076v1 
This figure "dns_stat3d.png" is available in "png" format from: http://arxiv.org/ps/astro-ph/0508076v1 
This figure "dns_strain_evol.png" is available in "png" format from: http://arxiv.org/ps/astro-ph/0508076v1 
This figure "les_energy_evol.png" is available in "png" format from: http://arxiv.org/ps/astro-ph/0508076v1 
This figure "les_nb20_energy_evol.png" is available in "png" format from: http://arxiv.org/ps/astro-ph/0508076v1 
This figure "les_nb_energy_evol.png" is available in "png" format from: http://arxiv.org/ps/astro-ph/0508076v1 
This figure "les_nb_q_sgs_evol.png" is available in "png" format from: http://arxiv.org/ps/astro-ph/0508076v1 
This figure "les_q_sgs_evol.png" is available in "png" format from: http://arxiv.org/ps/astro-ph/0508076v1 
This figure "les_stat3d_flame_speed.png" is available in "png" format from: http://arxiv.org/ps/astro-ph/0508076v1 
This figure "les_stat3d_locl.png" is available in "png" format from: http://arxiv.org/ps/astro-ph/0508076v1 